\title{
Genomic profiling of Nipah virus using NGS driven RNA-Seq expression data
}

\section{Md. Zakiul Hassan'†, Md. Shakil Ahmed1 ${ }^{1 *}$, Md. Marufuzzaman Khan², Mohammad Ahsan Uddin ${ }^{3}$, Fahmida Chowdhury ${ }^{1}$, Md. Kamruzzaman ${ }^{4}$}

${ }^{1}$ Infectious Diseases Division, International Centre for Diarrheal Disease Research, Bangladesh, Dhaka, Bangladesh; ${ }^{2}$ Department of Public Health, The University of Tennessee, Knoxville, Tennessee, USA; ${ }^{3}$ Department of Statistics, University of Dhaka, Dhaka, Bangladesh; 4Institute of Bangladesh Studies, University of Rajshahi, Rajshahi, Bangladesh; Md. Shakil Ahmed - Email: md.shakil@icddrb.org; tContributed equally; ${ }^{*}$ Corresponding author

Received December 28, 2019; Revised December 31, 2019; Accepted December 31, 2019; Published December 31, 2019

Abstract:

DOI: $10.6026 / 97320630015853$

Nipah virus (NiV) is an ssRNA, enveloped paramyxovirus in the genus Henipaveridae with a case fatality rate $>70 \%$. We analyzed the NGS RNA-Seq gene expression data of NiV to detect differentially expressed genes (DEGs) using the statistical R package limma. We used the Cytoscape, Ensembl, and STRING tools to construct the gene-gene interaction tree, phylogenetic gene tree and protein-protein interaction networks towards functional annotation. We identified 2707 DEGs (p-value <0.05) among 54359 NiV genes. The top-up and down-regulated DEGs were EPST1, MX1, IFIT3, RSAD2, OAS1, OASL, CMPK2 and SLFN13, SPAC977.17 using log2FC criteria with optimum threshold 1.0. The top 20 up-regulated gene-gene interaction trees showed no significant association between Nipah and Tularemia virus. Similarly, the top 20 down-regulated genes of neither Ebola nor Tularemia virus showed an association with the Nipah virus. Hence, we document the top-up and down-regulated DEGs for further consideration as biomarkers and candidates for vaccine or drug design against Nipah virus to combat infection.

Keywords: Nipah virus, NGS RNA-Seq, limma, Phylogenetic gene tree, Protein-protein interaction network

\section{Background:}

Nipah virus (NiV) is a stage III zoonotic pathogen from the family of Paramyxoviridae and a new genus from the Henipavirus [1]. Nipah virus was first discovered in a large encephalitis outbreak in Malaysia in 1998 [2-4]. Nipah virus outbreak has been recognized nearly every year in Bangladesh since 2001 and occasionally in neighboring India [5-9]. With the capacity of person-to-person transmission, high case fatality rate $(>70 \%)$ and no availability of treatment or vaccine, the World Health Organization included the Nipah virus among the 7 Blueprint list of priority diseases and effort for Nipah vaccine development is underway [10-12]. Genes are strongly involved in $\mathrm{NiV}$ infection in interferon response in endothelial cells. The chemokine CXCL10 (interferon-induced protein 10, IP-10) gene was identified among the top $10 \mathrm{up}$ regulated genes. The cellular functionality of CXCL10 is a generation of inflammatory immune response and neurotoxicity [13]. Arankalle, V. A et. al. performed the NiV whole-genome sequencing (18,252 nucleotides) from the lung tissue samples [14]. Detection of DEGs is an important branch of transcriptomics research in bioinformatics. RNA-sequencing (RNA-seq) is the modern Next Generation Sequencing (NGS) technology for genomic profiling of any bacteria, virus or pathogens and other causes of diseases. Identification of DEGs or transcripts associated with the specific trait of interest from the high dimension of transcriptomic data based on NGS RNA-Seq gene expression technique. Previously microarray technology had been used by biological and biomedical researchers for discovering the candidate genes and differentially expressed markers between two or more groups of interest. Additionally, this approach includes the identification of disease biomarkers that may be important in the diagnosis of the different types and subtypes of diseases, with several implications in terms of prognosis and therapy [15]. This sequence-based technology has created significant scope for studying the transcriptome and enabling a wide range of novel 


\section{BIOINFORMATION \\ Discovery at the interface of physical and biological Sceiencess}

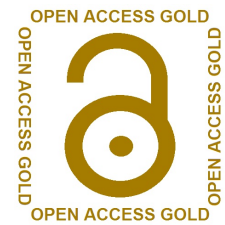

applications, including detection of alternative splicing isoforms [16-19], detecting novel genes, gene promoters, isoforms, and allelespecific expression [20]. RNA-seq uses NGS technology to sequence cDNA that has been derived from an RNA sample, and hence generates millions of short reads [21]. One important objective for RNA-seq is to identify DEGs under different conditions. Researchers typically target for differential expression analysis called "count matrix", where each row represents the gene, each column represents the sample, and each cell indicates the number of reads mapped to the gene in the sample [22]. A basic research problem in many RNA-seq analyses is the discovery of DEGs between different sample groups (e.g. healthy and disease). RNAseq analysis has some benefits over microarrays for DE analysis including wide dynamic range and a lower background level, and the chance to detect and quantify the expression of previously unknown transcripts [23]. Identification of differentially expressed genes from the large scale NGS RNA-Seq data and functional annotation of the Nipah virus were the key objectives of this study.

\section{Materials and Methods: \\ NGS RNA-Seq Microarray Gene Expression Dataset:}

We used Microarray gene expression of RNA-Seq data for molecular investigation of $\mathrm{NiV}$ infection. We collected the complete genome of selected pathogen from the National Centre for Biotechnology Information (NCBI). To analyze data we considered 7 (seven) different datasets with accession numbers in the GEO (Gene Expression Omnibus) database are as follows GSE32902, GSE23986, GSE93861, GSE18064, GSE12108, GSE69980, GSE89915 [13,24-29].

Table 1: Top 10 up regulated genes of Nipah virus

\begin{tabular}{llllllll}
\hline ID & adj. P Value & P Value & $\mathbf{t}$ & $\mathbf{B}$ & Log FC & Gene symbol & $\begin{array}{l}\text { Function } \\
\text { Status }\end{array}$ \\
\hline 426044 & 0.884 & $7.55 \mathrm{E}-05$ & 22.7 & -0.831 & 3.82 & EPSTI1 & Functional \\
82039 & 0.884 & 0.0001 & 20.9 & -0.853 & 4.52 & AI669006 & Non-functional \\
404052 & 0.884 & 0.000103 & 20.8 & -0.854 & 3.8 & MX1 & Functional \\
190107 & 0.884 & 0.000135 & 19.2 & -0.879 & 3.43 & IFIT3 & Functional \\
255017 & 0.884 & 0.000142 & 18.9 & -0.884 & 3.52 & RSAD2 & Functional \\
304097 & 0.884 & 0.000143 & 18.8 & -0.885 & 3.11 & OAS1 & Functional \\
60082 & 0.884 & 0.000155 & 18.4 & -0.893 & 3.44 & OASL & Functional \\
141045 & 0.884 & 0.000177 & 17.7 & -0.907 & 3.45 & Unknown & Unknown \\
442005 & 0.884 & 0.000186 & 17.4 & -0.913 & 2.95 & CMPK2 & Functional \\
123024 & 0.884 & 0.000189 & 17.4 & -0.915 & 4.52 & Unknown & Unknown \\
\hline
\end{tabular}

Download links for data are as follows:

[1] NCBI Gene Expression Omnibus (https://www.ncbi.nlm.nih.gov/geo/)

[2] Nipah Virus (https://www.ncbi.nlm.nih.gov/geo/query/acc.cgi?acc=GSE32902)

[3] Dengue virus type-3 (https://www.ncbi.nlm.nih.gov/geo/query/acc.cgi?acc=GSE23986)

[4] Ebola virus (https://www.ncbi.nlm.nih.gov/geo/query/acc.cgi?acc=GSE93861)

[5] Rift Valley Fever virus (https://www.ncbi.nlm.nih.gov/geo/query/acc.cgi?acc=GSE18064)

[6] Tularemia (https://www.ncbi.nlm.nih.gov/geo/query/acc.cgi?acc=GSE12108)

[7] Chikungunya (https://www.ncbi.nlm.nih.gov/geo/query/acc.cgi?acc=GSE69980)

[8] Zika virus (https://www.ncbi.nlm.nih.gov/geo/query/acc.cgi?acc=GSE89915)

Table 2: Top 10 down regulated genes of Nipah virus

\begin{tabular}{llllllll}
\hline ID & adj. P Value & P Value & $\mathbf{t}$ & $\mathbf{B}$ & $\log$ FC & Gene symbol & Function Status \\
\hline 213081 & 0.884 & 0.000223 & -16.5 & -0.934 & -4.27 & Unknown & Unknown \\
444022 & 0.884 & 0.000234 & -16.3 & -0.94 & -2.73 & LINC00244 & Non-functional \\
290080 & 0.884 & 0.000294 & -15.2 & -0.972 & -2.54 & AI806251 & Non-functional \\
493014 & 0.884 & 0.00035 & -14.5 & -0.998 & -3.1 & LOC101928812 & Non-functional \\
46044 & 0.884 & 0.000361 & -14.3 & -1.003 & -5.32 & SLFN13 & Functional \\
383031 & 0.884 & 0.000429 & -13.6 & -1.033 & -2.4 & LOC101929735/TRDV1 & Non-functional \\
74032 & 0.884 & 0.000529 & -12.8 & -1.071 & -2.51 & BQ272413 & Non-functional \\
475050 & 0.884 & 0.000592 & -12.4 & -1.094 & -2.07 & BM689708 & Non-functional \\
333045 & 0.884 & 0.000603 & -12.3 & -1.098 & -2.62 & PLAC4 & Non-functional \\
106012 & 0.884 & 0.000646 & -12 & -1.112 & -2.13 & AQP7P1 & Functional \\
\hline
\end{tabular}




\section{BIOINFORMATION \\ Discovery at the interface of physical and biological sciences}

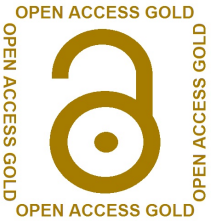

Statistical Analysis:

We performed the $\log 2$ transformation to analyze the RNA-Seq NGS gene expression data. The log transformation is the robust method for scaling and controlling the outliers of the dataset by uniform pattern. Linear model for microarray analysis (limma) is the efficient statistical tool integrated in $\mathrm{R}$ package for identification of DEGs from large scale expression data [30]. The log2FC (Fold Change) $=\log 2$ mean (Infected) $/$ mean (Normal) $\}$ method was used for selection of up and down-regulated DEGs [31]. The pvalue was calculated using a t-test statistic from the linear model. The Benjamini \& Hochberg (False discovery rate) was used for calculation of the adjusted p-value. All the statistical analysis was implemented using $\mathrm{R}$ statistical programming language software. For functional annotation and biological network of differentially expressed genes were analyzed using STRING, Ensembl and Cytoscape bioinformatics tools respectively. Figure 1 shows the

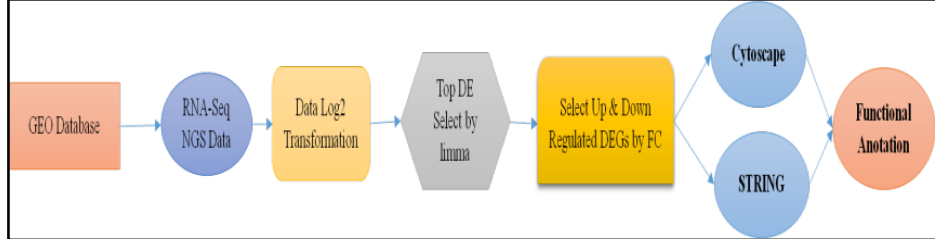

Figure 1: The schematic diagram of the work flow of the study of genomic profiling of Nipah virus

\section{Results and Discussion:}

Characteristics of Nipah virus dataset:

The row represents the gene and the column represents the RNA samples in the NGS RNA-Seq data matrix of Nipah virus (GEO ID: GSE32902). In this dataset four samples (two uninfected HUVEC and two NiV-infected HUVEC) and 54359 genes were included. work flow of the manuscript.

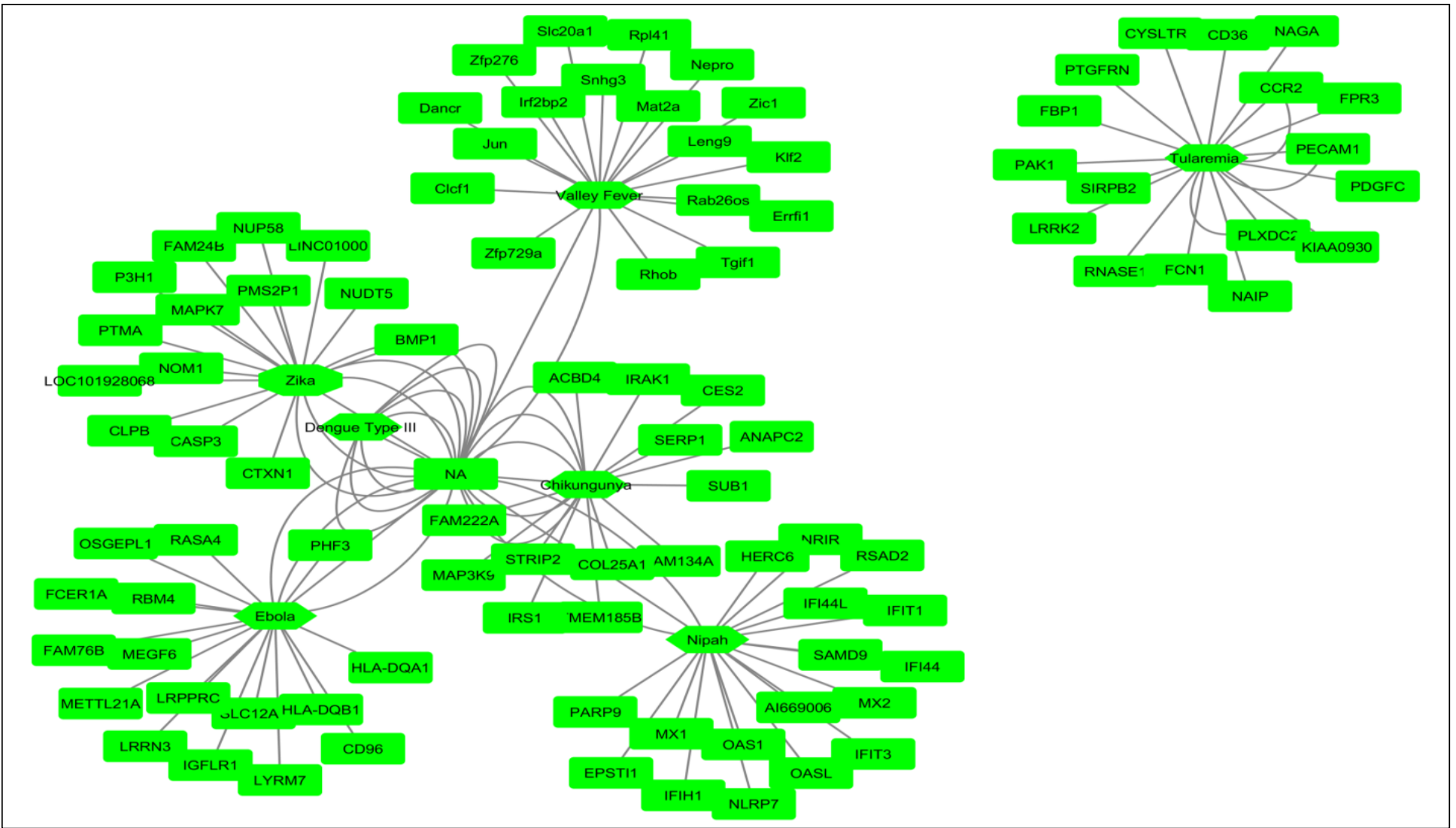

Figure 2: Association among zoonotic viruses including Nipah virus using gene-gene interaction network tree for top 20 up regulated DE genes 


\section{BIOINFORMATION}

\section{Discovery at the interface of physical and biological sciences}

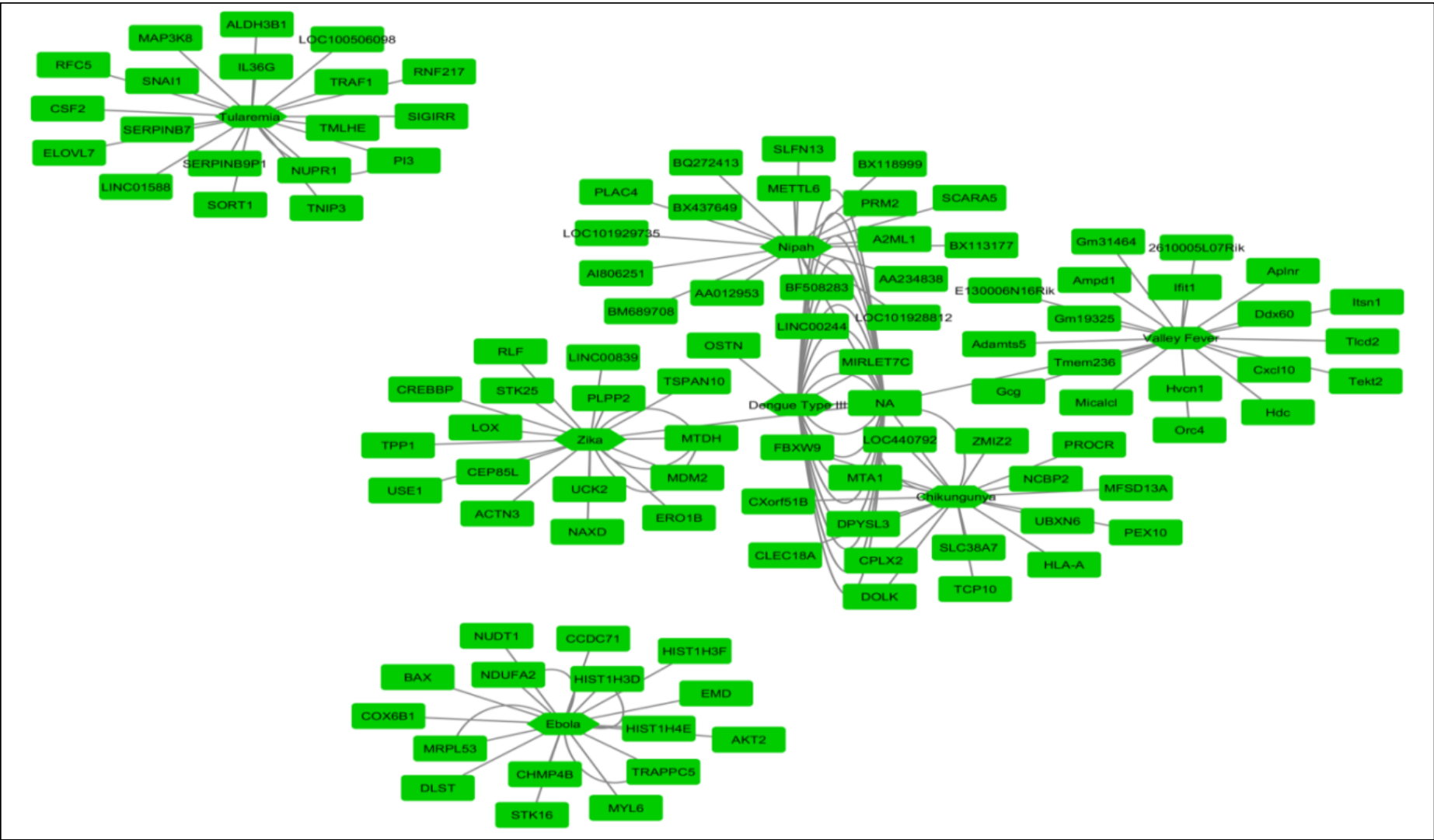

Figure 3: Association among zoonotic viruses including Nipah virus using gene-gene interaction network tree for top 20 down regulated DE genes

Table 3: Most predicted partners for top functional up- and down-regulated functional DEGs of Nipah virus

\begin{tabular}{|c|c|c|c|c|c|}
\hline \multirow{2}{*}{ Gene No. } & \multirow{2}{*}{ Target genes } & \multicolumn{3}{|c|}{ Most predicted partners } & \multirow{2}{*}{ P value } \\
\hline & & Gene symbol & Function & Score & \\
\hline (g1) & EPSTI1 (Epithelial stromal interaction 1; $410 \mathrm{aa}$ ) & $\begin{array}{l}\text { IFI44L (Interferon-induced protein 44-like; } \\
452 \text { aa) }\end{array}$ & $\begin{array}{l}\text { Exhibits a low antiviral activity against hepatitis } \\
\text { C virus }\end{array}$ & 0.736 & $<1.0 \mathrm{e}-16$ \\
\hline (g3) & $\begin{array}{l}\text { MX1 (Interferon-induced GTP-binding protein } \\
\text { Mx1; } 662 \text { aa) }\end{array}$ & $\begin{array}{l}\text { OASL(2/-5/-oligoadenylate synthase-like } \\
\text { protein) }\end{array}$ & $\begin{array}{l}\text { Does not have 2/-5/-OAS activity, but can bind } \\
\text { double-stranded RNA }\end{array}$ & 0.999 & $<1.0 \mathrm{e}-16$ \\
\hline (g4) & $\begin{array}{l}\text { IFIT3 (Interferon-induced protein with } \\
\text { tetratricopeptide repeats } 3 ; 490 \text { aa) }\end{array}$ & $\begin{array}{l}\text { IFIT1 (Interferon-induced protein with } \\
\text { tetratricopeptide repeats 1) }\end{array}$ & $\begin{array}{l}\text { Interferon-induced antiviral RNA-binding } \\
\text { protein }\end{array}$ & 0.999 & $<1.0 \mathrm{e}-16$ \\
\hline (g5) & $\begin{array}{l}\text { RSAD2 (Radical S-adenosyl methionine protein } \\
\text { 2; } 361 \text { aa) }\end{array}$ & $\begin{array}{l}\text { IFIT1 (Interferon-induced protein with } \\
\text { tetratricopeptide repeats 1) }\end{array}$ & $\begin{array}{l}\text { Interferon-induced antiviral RNA-binding } \\
\text { protein }\end{array}$ & 0.997 & $<1.0 \mathrm{e}-16$ \\
\hline (g6) & $\begin{array}{l}\text { OAS1 (OASL(2/-5/-oligoadenylate synthase 1; } \\
414 \text { aa) }\end{array}$ & $\begin{array}{l}\text { MX1 (Interferon-induced GTP-binding } \\
\text { protein Mx1) }\end{array}$ & $\begin{array}{l}\text { Interferon-induced dynamin-like GTPase with } \\
\text { antiviral activity }\end{array}$ & 0.997 & $<1.0 \mathrm{e}-16$ \\
\hline (g7) & OASL (2/-5/-oligoadenylate synthase-like protein) & $\begin{array}{l}\text { MX1 (Interferon-induced GTP-binding } \\
\text { protein Mx1) }\end{array}$ & $\begin{array}{l}\text { Interferon-induced dynamin-like GTPase with } \\
\text { antiviral activity }\end{array}$ & 0.999 & $<1.0 \mathrm{e}-16$ \\
\hline (g9) & CMPK2 (UMP-CMP kinase 2; 449 aa) & $\begin{array}{l}\text { RSAD2 (Radical S-adenosyl methionine } \\
\text { domain-containing protein 2) }\end{array}$ & $\begin{array}{l}\text { Interferon-induced inron-sulfur (4FE-4S) cluster- } \\
\text { binding activity }\end{array}$ & 0.967 & $2.62 \mathrm{e}-08$ \\
\hline \multicolumn{6}{|c|}{ Top down-regulated functional DEGs of Nipah virus } \\
\hline (g5) & SLFN13 (Schlafen family member 13; 897 aa) & EVA1B(Eva-1 homolog B; 165 aa) & Belongs to the EVA1 family & 0.474 & 0.0613 \\
\hline (g10) & $\begin{array}{l}\text { SPAC977.17 (Uncharacterized membrane protein } \\
\text { C977.17; } 598 \text { aa) }\end{array}$ & $\begin{array}{l}\text { SPAC29B12.11c.1 (UPF0664 stress-induced } \\
\text { protein C29B12.11c) }\end{array}$ & Human WW domain binding protein-2 ortholog) & 0.882 & 0.0389 \\
\hline
\end{tabular}




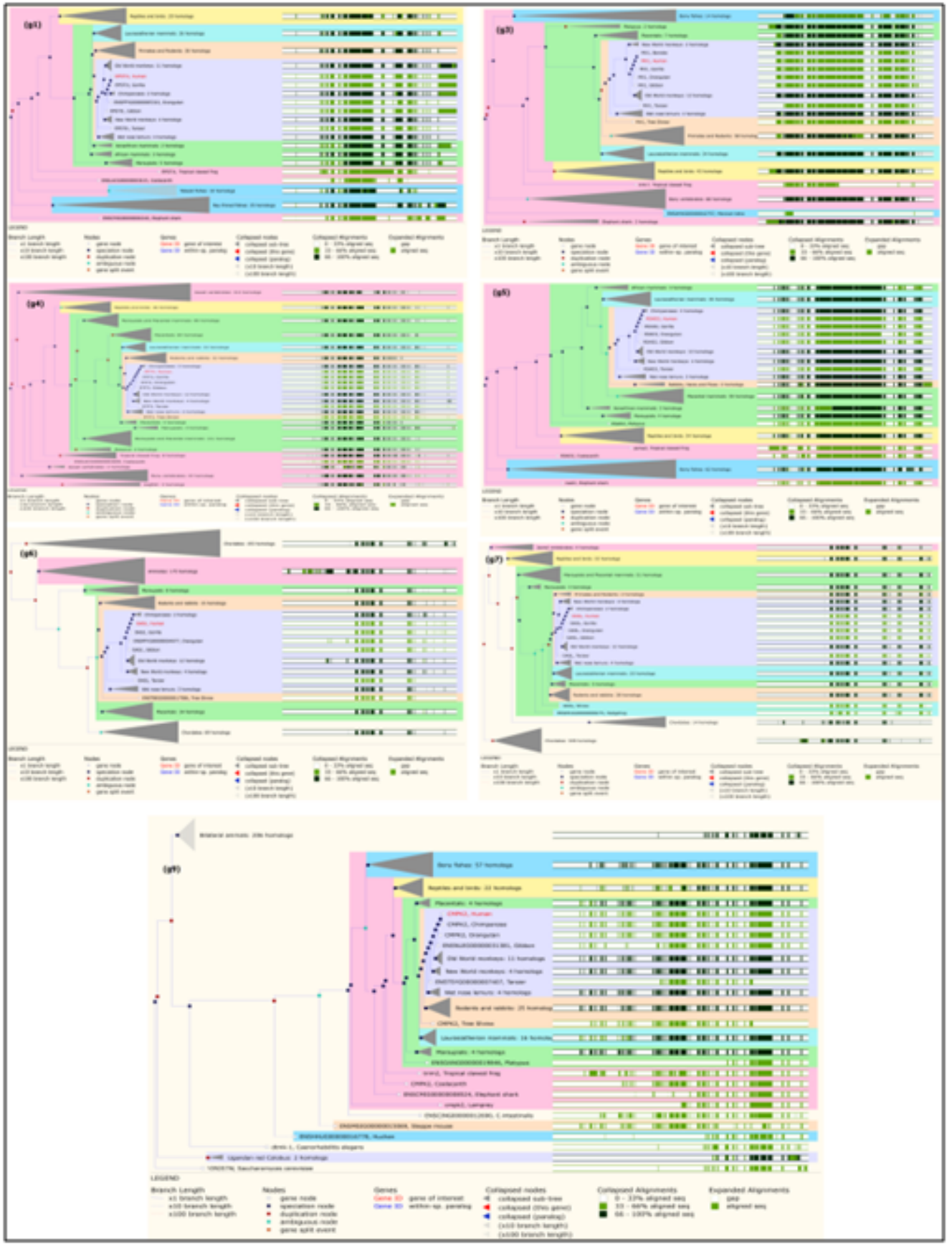

Figure 4: Gene tree for genomic classification of top up regulated seven functional DE genes: (g1) EPST1; (g3) MX1; (g4) IFIT3; (g5) RSAD2; (g6) OAS1; (g7) OASL; (g9) CMPK2 


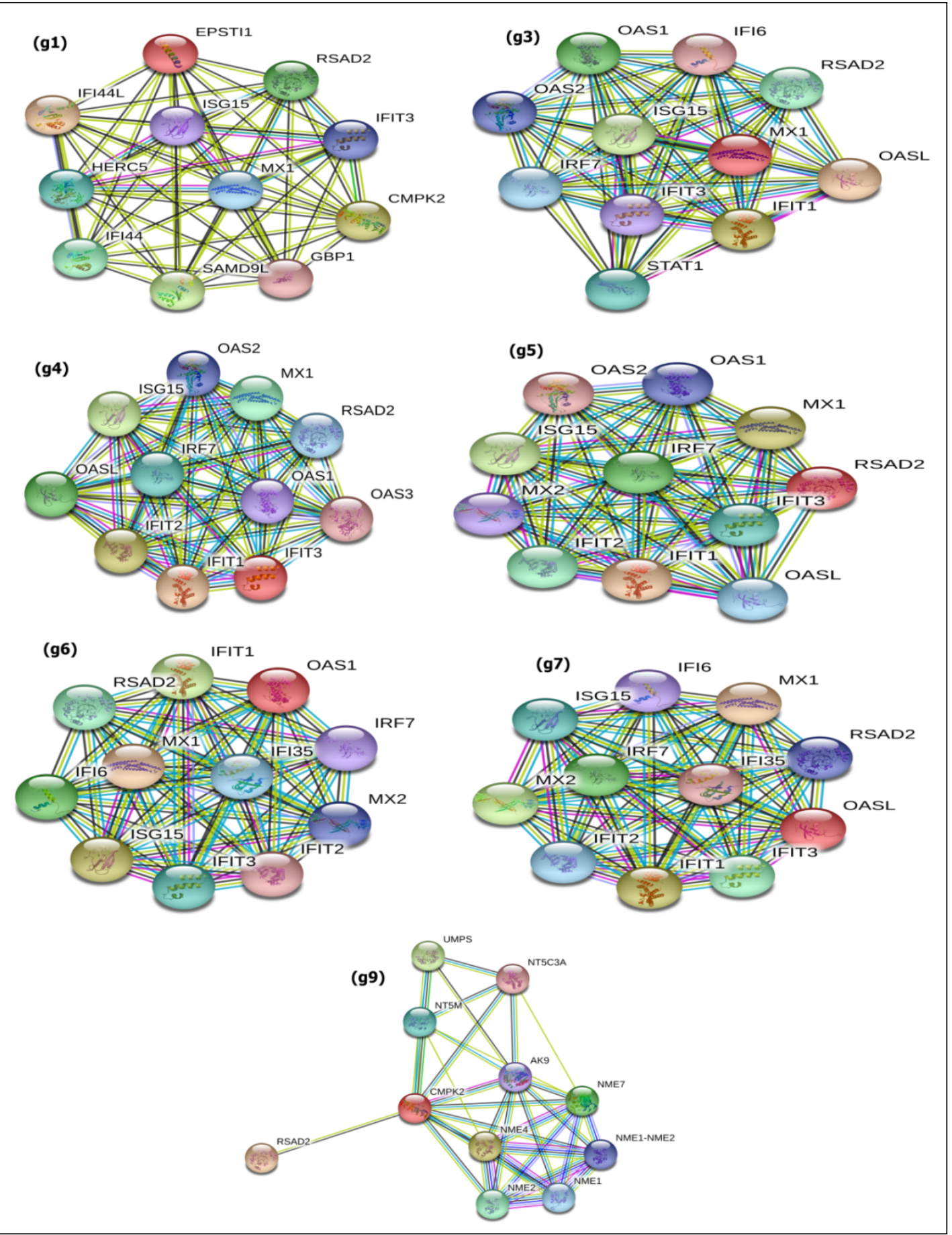

Figure 5: Protein-protein interaction network for top up regulated seven functional DE genes (red color node is the target gene node): (g1) EPST1; (g3) MX1; (g4) IFIT3; (g5) RSAD2; (g6) OAS1; (g7) OASL; (g9) CMPK2 


\section{BIOINFORMATION}

\section{Discovery at the interface of physical and hiological sciences}

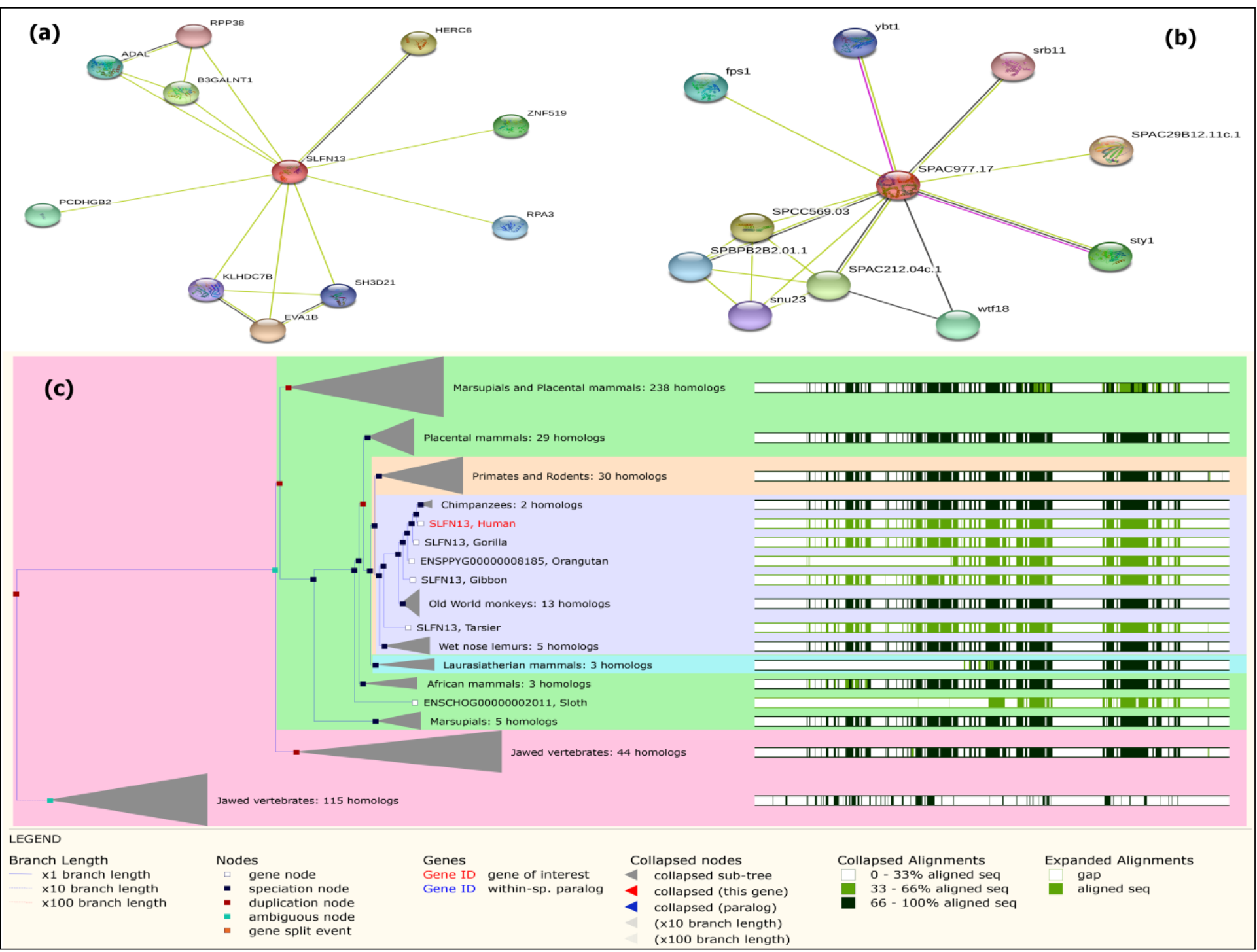

Figure 6: Protein-protein interaction network and phylogenetic gene tree for top down regulated two functional DE genes. (a) PPI network of SLFN13 (b) PPI network of SPAC977.17 (C) phylogenic gene tree of SLFN13

\section{Differentially Expressed Genes (DEGs) Detection:}

We used t-test statistic to calculate the $\mathrm{p}$-values for each gene (54359) of Nipah virus dataset. We used a cut of point less than 0.05 at a 5\% level of significance to select the DEGs. We identified 2707 DEGs with this cut of value. Similarly, for Chikungunya virus 1544 DEGs out of 45220 genes were identified using a $p$ value $<0.05$ cut of point. We identified 1200 DEGs (p value $<0.05$ ) of 33297 genes of the Dengue type III virus. The Ebola Virus RNA-Seq dataset included 41000 genes and of these 20211 genes were detected as DEGs using a $\mathrm{p}$ value $<0.05$ in $\mathrm{t}$-test statistic. With the same $\mathrm{p}$-value cut of point
$(<0.05)$, we identified 14470 DEGs of 54675 genes for Tularemia (Francisella tularensis), 6666 DEGs of 45101 genes for Rift valley fever virus and, 688 DEGs of 43376 genes for Zika virus.

\section{Up and Down Regulated DEGs Detection:}

We used the $\log 2$ fold change or a cutoff at 0.5 for down and 1/2 for up-regulated genes and selected genes under/above thresholds. A $\log 2 \mathrm{FC}$-value close to 0.5 corresponds to equally expressed (EE) genes, while down- and up-regulated genes have log2FC-values close to 0 and 1 correspondingly [20]. In this study, $\log 2 \mathrm{FC}$ cut of 


\section{BIOINFORMATION

value 1 was used for up- and down-regulated DEGs selection for all viruses except dengue type III (log2FC cut of 0.5$)$. With the $\log 2 \mathrm{FC}$ cut of thresholds, 834 up- $(\log 2 \mathrm{FC}>1.0)$ and 1873 down-regulated $(\log 2 \mathrm{FC} \leq 1.0)$ DEGs in Nipah virus, 743 up- and 801 down-regulated DEGs in Chikungunya, 8 up- $(\log 2 \mathrm{FC}>0.5)$ and 1192 downregulated $(\log 2 \mathrm{FC} \leq 0.5)$ DEGs in Dengue type III, 3271 up- and 16940 down-regulated DEGs in Ebola virus, 251 up- and 14219 down-regulated DEGs in Tularemia, 1204 up- and 5462 downregulated DEGs in Rift valley fever virus, and 338 up- and 350 down-regulated DEGs in Zika virus.

\section{Gene-Gene Interaction Network:}

We used Cytoscape (https://cytoscape.org/) bioinformatics tool to construct a gene-gene interaction network. Based on the calculated p-value, we selected top 20 up- and down-regulated genes ( $p$-value $<0.000481$ and $\log 2 \mathrm{FC}>1$ ) for 7 viruses (Nipah, Chikungunya, Dengue type III, Ebola, Tularemia, Valley fever, Zika) ranked according to increasing order for each gene. The gene-gene interaction tree of the top 20 up-regulated DEGs among the 7 viruses showed that the Tularemia virus had no association with the other 6 viruses including the Nipah virus and, it represents a separate gene tree. The other five viruses, however, showed that a significant association with the Nipah virus and unknown gene symbol (NA; not available) and a strong network was seen among these viruses (Figure 2). From the gene-gene interaction tree of the top 20 down-regulated DEGs, we found that the Ebola and Tularemia virus showed no association with the other five viruses and showed a separate pattern. Nipah virus was strongly associated with Chikungunya, Dengue type III, Valley fever, and Zika virus. The unknown gene symbol (NA) showed a strong association with Nipah, Chikungunya, Dengue type III, Rift Valley fever, and Zika viruses (Figure 3).

\section{Phylogenetic Gene Tree for top DE genes of Nipah Virus:}

We selected the top 10 up-regulated genes using ranking p-value. Of 10, 7 were functional: EPST1 (g1), MX1 (g3), IFIT3 (g4), RSAD2 (g5), OAS1 (g6), OASL (g7), CMPK2 (g9) and 3 were non-functional genes (Table 1). We used the Ensembl (https://asia.ensembl.org/index.html) online bioinformatics tool for the construction of the phylogenetic gene tree of top upregulated functional DEGs. We found that EPST1, Human gene were similar cluster group with EPST1, Gorilla (Figure 4 (g1)); MX1, Human gene highly similar cluster group with MX1, Gorilla (Figure 4 (g3)); IFIT3, Human gene highly similar cluster group with Chimpanzees: 2 homologs (Figure 4 (g4)); RSAD2, Human gene highly similar cluster group with Chimpanzees: 2 homologs (Figure 4 (g5)); OAS1, Human gene highly similar cluster group with Chimpanzees: 2 homologs (Figure 4 (g6)); OASL, Human gene highly similar cluster group with Chimpanzees: 2 homologs (Figure 4 (g7); CMPK2, Human gene highly similar cluster group with CMPK2, Chimpanzee (Figure 4 (g9)) from the phylogenetic gene tree (Figure 4). The functional down-regulated DEGs SLFN13, Human highly similar cluster group with Chimpanzees: 2 homologs from the phylogenetic gene tree (Table 2 and Figure 6(c)).

\section{Protein-protein Interaction Network:}

We used the STRING (https://string-db.org/) online bioinformatics tool for protein-protein interaction (PPI) networks functional enrichment analysis. Top up-regulated seven functional EPST1 (g1), MX1 (g3), IFIT3 (g4), RSAD2 (g5), OAS1 (g6), OASL (g7), CMPK2 (g9) DEGs of Nipah virus were highly interacting proteins by score with IFI44L, OASL, IFIT1, IFIT1, MX1, MX1, and RSAD2 respectively. All the PPI network enrichment was statistically significant ( $p$ value $<0.05$ ) at a $5 \%$ level of significance (Figure 5). All top interacting genes were interferon (INF) induced proteins that exist in the antiviral functional pathway (Table 3). Previous analysis by Nicole B et al. who performed the initial genomic profile of virus-induced innate immune response in Pteropus vampyrus bats, a known reservoir of the Nipah virus. The study found that henipavirus IFN antagonist mechanisms are likely active in bat cells [32-33]. Similarly the top down-regulated functional DEGs SLFN13 and SPAC977.17 were best interaction with EVA1B (0.474; p value=0.0613) and SPAC29B12.11c.1 (0.882; p value $=0.0389)$ proteins according to score and p-value $(<0.05)$ (Table 3; Figure 6 (a) and (b)).

\section{Conclusions:}

We used the statistical R package limma to analyze the NGS RNAseq data to detect DEGs (biomarker) of the Nipah virus for application in combat and care of the disease. We identified 2707 DEGs (p-value <0.05) among the 54359 genes of the virus. We report 834 up-regulated and 1873 down-regulated DEGs estimated by the $\log 2 \mathrm{FC}$ approach at threshold value 1.0. This data will help in the selection of biomarkers and vaccine targets against the virus.

\section{Acknowledgements:}

This work did not receive any external funding. icddr, $b$ is grateful to the Governments of Bangladesh, Canada, Sweden, and the United Kingdom for providing core/unrestricted support.

\section{References:}

[1] Eaton BT et al. Nat Rev Microbiol. 2006 4: 23.[PMID: 16357858]

[2] "WHO Nipah Virus (NiV) Infection" 18 April 2018.

[3] Stone R Science 2011 331: 1128. [DOI: 10.1126/science.331.6021.1128]

[4] Griffin BD et al. Sci Rep. 2019 9:11171 [PMID: 31371748]

[5] Luby PS et al. Emerging Infectious Diseases 2009 15: 1229. [PMID: 19751584]

[6] Hassan MZ et al. Emerging infectious diseases. 2018 24:15. [PMID: 29260663] 


\section{BIOINFORMATION \\ Discovery at the interface of physical and biological sciences}

[7] Nikolay B et al. New England Journal of Medicine. 2019 380:1804. [PMID: 31067370]

[8] Yadav PD et al. Am J Trop Med Hyg. 2012 87:576. [PMID: 22802440]

[9] Thomas B et al. Indian J Community Med. 2019 44:383. [PMID: 31802805]

[10] https://www.who.int

[11] https://www.ologybio.com

[12] McLean RK et al. Frontiers in Veterinary Science. 2019 6:16. [PMID: 30778392]

[13] Mathieu Cet al. PLoS One. 2012 7:e32157. [PMID: 22393386]

[14] Arankalle VA et al. Emerging infectious diseases. 2011 17:907 [PMID: 21529409]

[15] Dillies MA et al. Briefings in Bioinformatics. 2012 14:671. [PMID: 22988256]

[16] Wang et al. Nature. 2008 456:470.[PMID: 18978772]

[17] Lo MK et al. J Infect Dis. 2019. [PMID: 31751453]

[18] Ravichandran L et al. J Cell Biochem. 2018 [PMID: 30417438]

[19] Jensen KS et al. PLoS One. 2018 13:e0199534. [PMID: 29920552]

[20] Landau et al. PLoS ONE. 2013 8:e81415.

[21] Soneson C \& Delorenzi MA. BMC Bioinformatics. 2013 14:91.
[22] Tang M et al. BMC Bioinformatics. 2015 16:361. [PMID: 26538400]

[23] Oshlack A et al. Genome Biology.2010. 11:220.

[24] Silveira GF et al. Journal of virology. 2011 85:5374. [PMID: 21450836] [PMCID: PMC3094967]

[25] Caballero IS et al. BMC genomics. 2016 17: 707. [PMID: 27595844] [PMCID: PMC5011782]

[26] do Valle TZ et al. The Journal of Immunology. 2010 185:6146. [PMID: 20937849]

[27] Butchar JP et al. PloS one. 2008 3: e2924. [PMCID: PMC2488368] [PMID: 18698339]

[28] Karlas A et al. Nature communications. 2016 7:11320. [PMID: 27177310] [PMCID: PMC4865845]

[29] Kozak R et al. Viruses. 2017 9:297. [PMID: 29036922] [PMCID: PMC5691648]

[30] http://www. bioconductor.org/

[31] Dembele D et al. BMC Bioinformatics. 2014 15:14. [PMID: 24423217]

[32] Glennon NB et al. J Virol. 2015 89:7550. [PMID: 25972557] [PMCID: PMC4505658]

[33] Wacharapluesadee S et al. J Virol Methods. 2007 141: 97 .[PMID: 17184850]

Edited by $\mathbf{P}$ Kangueane Citation: Hassan et al. Bioinformation 15(12): 853-862 (2019)

License statement: This is an Open Access article which permits unrestricted use, distribution, and reproduction in any medium, provided the original work is properly credited. This is distributed under the terms of the Creative Commons Attribution License

\section{Articles published in BIOINFORMATION are open for relevant post publication comments and criticisms, which will be published immediately linking to the original article for FREE of cost without open access charges. Comments should be concise, coherent and critical in less than 1000 words.}




\section{BIOINFORMATION}

Discovery at the interface of physical and biological sciences
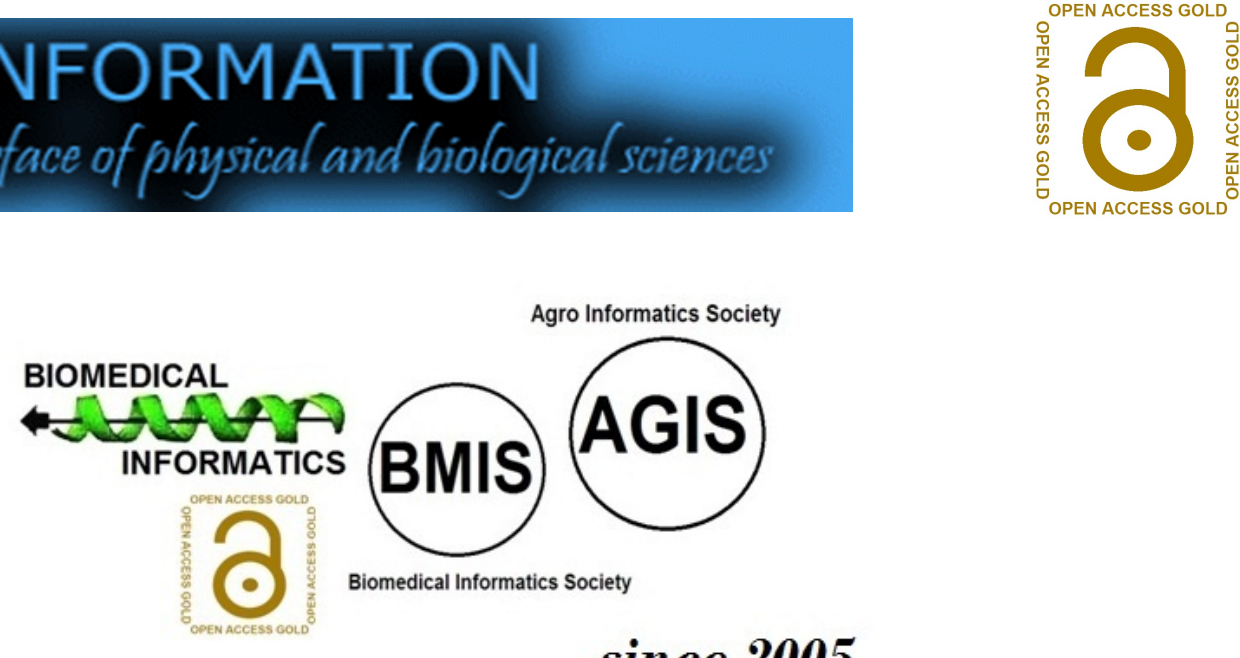

since 2005

\section{BIOINFORMATION}

Discovery at the interf face of physical and biological sciencess

\section{indexed in}
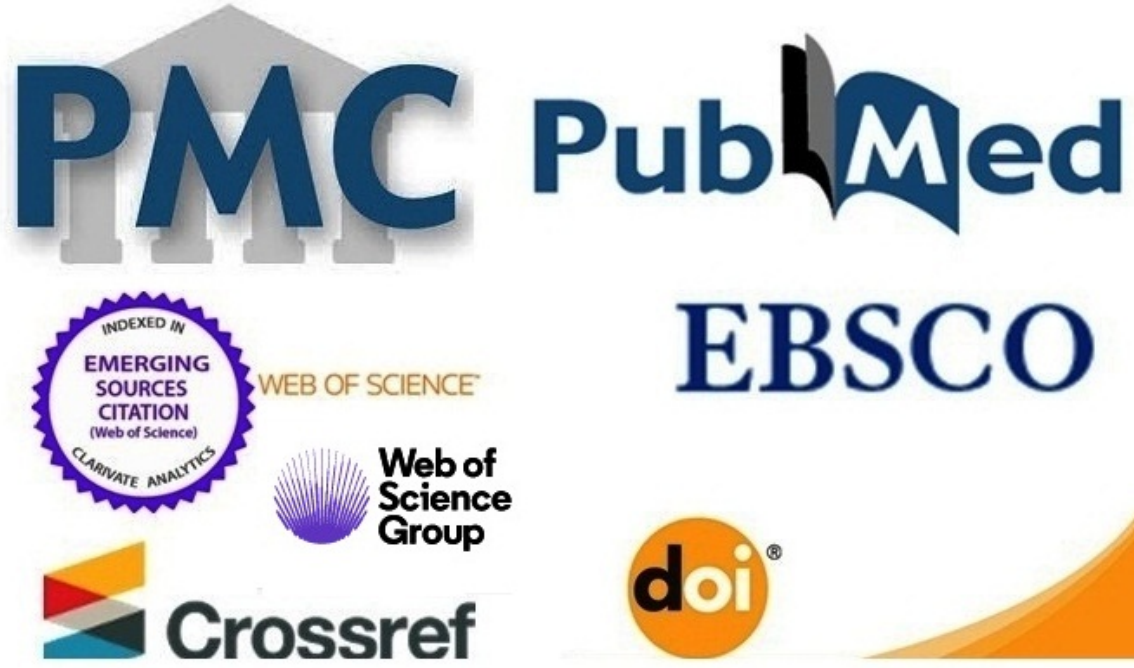

Web of

Science

Group

ResearchGate
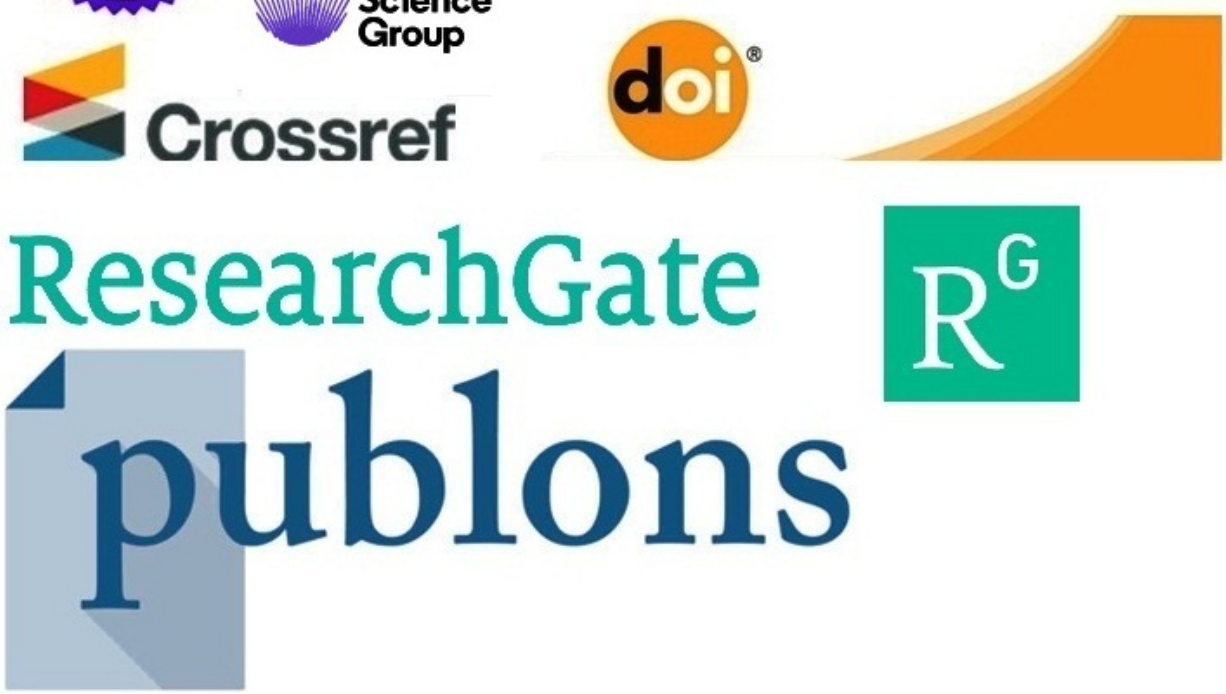